\title{
Wolf-Dieter Narr
}

\section{Das nicht so neue Tandem: Gewalt und Globalisierung}

Die Kritik der Gewalt ist die Philosophie ihrer Geschichte (Walter Benjamin)

\section{11.9.2001 Nackte Gesten der Gewalt}

Gewalt erscheint abrupt. Bricht ein. Bricht ab. So auch spektakulär in all ihren Dimensionen am 11.9.2001. Erst die besondere Kombination dieser Dimensionen macht die unmittelbaren und die bis heute erkenntlichen mittelbaren Wirkungen verständlich. Die Tötung von Tausenden von Menschen auf einen Schlag. Die Zerstörung von architektonisch eminenten Symbolen ökonomischer und politischer Macht. Der Standort dieser nicht nur als Symbole unübersehbaren Bauklötze im Land des Welthegemons. Dort schienen die Sicherheit der Herrschaft, ihres Territoriums und ihrer Leute geradezu absolut. Die Art der Zerstörung, die einem technologischen Bumerang glich. Entwickeltste Technik, vom Ort entwickeltster Technik mit best ausgebildeten Technikern losgeflogen gegen Gebäude, die nur mit entwickeltster Technik gebaut und in Gang gehalten werden konnten. Mehrere macht- und technikfeste Vorstellungen kollabierten auf einmal. Das bleibende Leid, dass Tausende von ganz individuellen Personen umgekommen sind, durch die zerstörten Gebäude im Tod zwangskollektiviert, erklärt, nüchtern gesprochen, die anhaltenden Wirkungen nicht.

Gerade weil die Gewaltakte vom 11. September von symbolischer Bedeutung so überladen sind, die sich von ihren „realen“ Effekten nicht säuberlich scheiden lässt, gerade darum bewirkten sie keine Denkpause, die angemessenes Reagieren wahrscheinlicher gemacht hätte. Sie öffneten die Augen nicht. Sie erzeugten blinde Reaktionen jedenfalls dort, wo die herrschende, amerikanisch gekrönte symbolische Gewalt am stärksten ihrerseits symbolisch getroffen scheint. Im amerikanischen, ostwärts von den übrigen NATOLändern geteilten Anspruch auf eine ungefährdete weltweite Dominanz, die unverändert und aller globalen Durchdringung zum Trotz im Anspruch territorialer Integrität unverändert zum Ausdruck kommt. Ein Anspruch der unter anderem die Rede Bushs vom 20.9. durchzieht: Amerika kann (!) nicht verletzt werden. 
Darum die neue Welt in alten Formeln: Gut und Böse; Feind und Freund; Entweder/Oder. Darum der vom eigenen Sicherheitsglauben durchdrungene eigenartig postmoderne Kreuzzug gegen den je und je neu zu bestimmenden Terrorismus, der als abstrakt „schlafende“ Gefahr, überall und jederzeit konkret werden kann. Und alle Staaten und ihre Staatsleute, die sich diesem Kreuzzug nicht anschließen, werden ihrerseits terroristisch verdächtig. Nicht umsonst scharen sie sich eilfertig, in ihren eigenen Sicherheitsinteressen konform, „solidarisch“ um die zugleich kapitalistische, militärische und kulturelle Führungsmacht. Darum tritt noch mehr als anhaltend zuvor die globale, jedoch höchst regional orientierte Geopolitik an die Stelle der ohnehin immer schon primär nur instrumentell eingesetzten Menschenrechte und einer ihnen entsprechenden Politik. Eine „Politik“ der antiterroristischen Gleichschaltung macht sich breit, die neue „Freunde“ im Rahmen der grotesken Herrschafts-"Solidarität" schafft und sonstige Gewaltakte aller Art wie den russischen Krieg gegen Tschetschenien zu sicherheitsverständlichen Aktionen erhebt.

Das ist denn die wahre Katastrophe nach der Katastrophe des 11.9.2001. Dass keine Gewaltpause eingetreten ist. Dass der 11.9. von der herrschenden USA und ihren westlichen Verbündeten primär - die BRD und ihre umgehend militärisch behelmten Staatsleute an der Spitze - als Gelegenheit zum erweiterten Gegenschlag begriffen worden ist und ergriffen wird. Um noch weitere Teile dieser Welt zu direkten und indirekten Protektoratsgebieten zu machen. Um in äußerer und damit wieder enger verbundener innerer Sicherheit die eigenen ökonomisch wohlständischen Interessen in riskanter werdenden Zeiten vorausgreifend zu schützen.

\section{Der Schein unmittelbarer Gewalt, der den Gewaltzyklus am Laufen hält}

„Urplötzlich bricht die Gewalt herein, ohne jede Vorwarnung.“ So fasst Wolfgang Sofsky erste individuelle und kollektive Gewalterlebnisse, die darum meist nicht zu Erfahrungen werden, also zu reflektierten Erlebnissen, aus denen gelernt werden könnte.

„Es ist als stürzte sie vom Himmel hernieder. Noch ehe sich jemand besinnen kann, ist es geschehen. Im Moment der Explosion sind die Menschen überwältigt. Sie haben keine Chance zur Gegenwehr, zur Deckung, zur Flucht. Die Bombe lässt keine Zeit zur Reaktion. Ein ohrenbetäubender Knall - von manchem ist nicht einmal mehr eine Spur erkennbar“" (Sofsky 2001).

Darum, weil Gewalt unmittelbar erscheint, sind unmittelbare, ihrerseits gewaltmimetische Reaktionen üblich. Gewalt, Gegengewalt. Gewalt, so scheint es, kann nur durch Gewalt begegnet werden. Für diese geradezu jahrtausend-, wenn nicht menschheitsgeschichtlich alte Devise spricht die doppelte Eigenart physischer Gewalt. Diese Gewalt wirkt unmittelbar. Ich werde verletzt. Ich 
werde festgenommen. Diese unmittelbare Wirkung ergibt sich aus der Totalität physischer Gewalt. Sie erfasst die ganze Person. Sie signalisiert die Verletzlichkeit des Menschen; sie ist verbunden mit Todesdrohung und Tötung. Das macht den geradezu absoluten Charakter dieser Gewalt aus. Physische Gewalt ist darum das Gegenteil in der Regel sprachlich vermittelter Verständigung bzw. sprachlich ausgetragener Konflikte. Sie bedeutet jeweils das Ende von Politik.

Umso wichtiger ist es zu lernen - ein Lernergebnis, das die unendliche Geschichte des Gewaltzyklus geradezu aufnötigt -, dass individuelle und kollektive Gewalt in Ausnahmen nahezu ausschließender Regel hochgradig vermittelte menschliche Äußerungsformen darstellen. Gewalt wird nicht einfach 'spontan' geübt mit rätselhaftem Ursprung. Gewalt hat vielmehr eine kürzere oder längere Vorgeschichte. Sie ist, obgleich offensichtlich menschenmöglich, freudianisch gesprochen im Triebhaushalt des Menschen angelegt, das Ergebnis einer verschieden langen und verschiedenartigen Kette von Bedingungen, deren „Syndrom“, deren Zusammenkommen schließlich einen Gewaltakt zustandebringt.

Überall dort, wo nicht unmittelbare Gefahr unmittelbare Schutzreaktionen legitimiert - ein allgemein anerkanntes individuelles und nicht ganz so unproblematisches kollektives, etwa staatsbezogenes Recht -, ist es deshalb dringlich, so man besagten Gewaltzyklus anhalten, ja durchbrechen will, die jeweilige Vermitteltheit von Gewalt aufzudröseln, um auf die Umstände ihrer Erzeugung einwirken zu können.

\section{Sparsame Notiz zum Gewaltbegriff}

Den Begriff der Gewalt gibt es nicht. Es kann ihn angesichts des geschichtlich vermittelten Charakters von Gewalt und der Fülle möglicher Gewalterscheinungen nicht geben. Insofern gilt Max Webers Kennzeichnung für den verwandten Machtbegriff analog: Er ist „soziologisch amorph“.

Gestalt gewinnt Gewalt erst, wenn sie institutionell gefasst wird. Etwa, die Moderne überragend, im „Monopol legitimer physischer Gewaltsamkeit“, das Weber gemäss im Sinne des ihm ureigenen Instruments den modernen Staat vor allen anderen Organisationen auszeichnet. Indes: auch wenn das staatliche Gewaltmonopol institutionell und funktional vergleichsweise klar gefasst werden kann, wie es jeweils organisiert ist, ja, schon ob es gegeben ist, wie es eingesetzt wird und was es bedeutet, ist unvermeidlicherweise hochgradig umstritten. Deswegen ist es problematisch - eine Schwäche nicht nur der Jurisprudenz, sondern auch der meisten sozialwissenschaftlichen Studien, vom „Gemeinverstand“ ganz zu schweigen -, das staatliche Monopol physischer Gewalt qua Verfassung und Sozialisation, jedenfalls in „westlichen“ Breiten, so selbstverständlich als legitim vorauszusetzen, dass seine dauernde Gewalt- 
förmigkeit und die in ihm gerade qua Monopol steckenden Gefahren allenfalls wahrgenommen werden, wenn es zu skandalösen „Übergriffen“ kommt. Dass Gewalt vom staatlichen Monopol dauernd präsent gehalten wird und die gesamte Politik des jeweiligen Staatsgebildes in all seinen Äußerungsformen bestimmt, wird geflissentlich „naiv“ übersehen. Staatliche Gewalt wird meist gar nicht als Gewalt diskutiert. Gewalt wird zur illegitimen Äußerung „Privater“. Analog wird das Problem des seinerseits soziologisch amorphen Terrorismus als selbstverständlich vom staatlichen Gewaltmonopol und denen, die über dasselbe verfügen, zu bekämpfende „Privatangelegenheit“ behandelt. Es sei denn abnorme „Schurkenstaaten“ betrieben solchen Terrorismus mit.

Die Schwierigkeiten, Gewalt „auf den Begriff zu bringen“, gründen jedoch nicht primär in den Chamäleonfarben - „öffentlich“, „privat“ - physischer Gewalt oder ihren diversen Erscheinungsformen und Wirkungen. Die Schwierigkeiten resultieren vor allem daraus, andere denn physische Erscheinungen von Gewalt genauer zu fassen, vor allem aber aus dem Umstand, Gewalt so zu begreifen, dass ihre Genesis mit in den Blick käme.

Johan Galtung hat vor Jahrzehnten den seinerzeit heftig diskutierten Begriff „strukturelle Gewalt“ eingeführt. Mit Hilfe dieses Begriffs wollte er auf Verhältnisse/Institutionen/Strukturen/Maßnahmen/Verhaltens- und Aktionsformen aufmerksam machen, die von Menschen interessiert geschaffen und betrieben, andere Menschen systematisch schädigen: sie mangelhaft ausbilden zu lassen; sie arbeitslos zu halten; sie sozial negativ zu privilegieren; sie hungern zu machen, sie zu töten. Der große Vorzug des Begriffes „strukturelle Gewalt" war und ist, dass soziale Umstände ihres schicksalhaften Anscheins entkleidet werden. Die nackten Interessen treten zu Tage, die solche Umstände durch jeweils herrschende und davon profitierende Menschengruppen oder ganze Länderkomplexe etwa im Vergleich der Nord- zur Südhälfte der bewohnten Erde anhaltend erzeugen. In diesem Sinne erzählte der kosmopolitische Norweger Galtung des Dänen Andersen Märchen Von des Kaisers neuen Kleidern verändert neu. Letzteres sollte ohnehin täglich zur Pflichtlektüre aller Orten gemacht werden. Dann hätte das herrschafts- und herrschaftspersonenromantische Glotzen ein Ende. Die große Schwäche des Begriffs „strukturelle Gewalt“ bestand und besteht darin, dass sein Gegenbegriff, nämlich gewaltfreie gesellschaftliche Umstände, zu vage geraten ist. Außerdem droht der Gewaltbegriff alle eigenen Konturen zu verlieren und wird unspezifisch mit all den Erscheinungen und Faktoren deckungsgleich, die Ungleichheit(en) unter den Menschen erzeugen. Dann lässt er nichts Eigenständiges mehr begreifen, wird also ein Un-Begriff.

Ich habe den seinerzeit auch verfassungsschützerisch diskriminierten und sozusagen regierungsoffiziell unterdrückten Begriff „strukturelle Gewalt“ wieder erinnert, weil es mir darum zu tun ist, den Blick auf die Vermitteltheit von 
physischer Gewalt zu lenken; aktuell dessen zu erinnern, was als Terrorismus weltweit, konzentriert auf Afghanistan, morgen vielleicht auf den Irak, übermorgen ... westkriegssolidarisch bekämpft wird. Ich will auf Faktoren aufmerksam machen, die genetisch und funktional im Kontext der Globalisierung, in und durch die Eigenarten dessen, was da Globalisierung heißt - den PROKLA-Leserinnen und Lesern nicht mehr zu erläutern - kollektive Gewalt bedingen, die ihrerseits mit Gewalt bekriegt werden mit dem weiten Mantel staatlich-westlich-zivilisatorischer Legitimation bedeckt. Die gegenwärtige, weltdurchhallende Parole „Haltet die Terroristen“, die bis in feine innenpolitische Ritzen dringt, lenkt, so meine nicht originelle Behauptung, von den dynamisch sich entwickelnden Bedingungen ab. Diese Umstände werden nicht zuletzt ,west"-geschaffen. Sie haben zur Folge, dass divers gebildete Staudämme der Aggression noch und noch aufgefüllt werden, bis sie hier oder dort, so oder so überlaufen bzw. bersten. Die Staudämme werden hierbei oft von Staaten und Quasistaaten gebildet. Und unter diesen gestauten Aggressionen finden sich Millionen und Aber-Millionen von Menschen, die ihren elenden Verhältnissen nicht einmal aggressiv oder handelnd durch Migration begegnen können. Sie gehen im weltkapitalistisch wachsenden Meer abstrakt allgemeiner „Menschenrechte“ jämmerlich zugrunde.

Meine folgende Skizze wird also über die dem globalen Kapitalismus und seinen Herrschaftsprofiteuren innewohnende vermittelte Gewalt im Sinne der Produktion gewaltgeneigter Bedingungen handeln. Hierbei ist darauf zu achten, dass manche der genannten Bedingungen an sich selber schon Menschen Gewalt antun, allerdings ohne als Gewalt unmittelbar in Erscheinung zu treten.

\section{Historisch-gegenwärtige Notiz: Staatsgewalt und kapitalistische Volkswirtschaft oder Nationalökonomie}

a) Die europäische angelsächsische Moderne ist durch einen Jahrhunderte dauernden (und nie abgeschlossenen) Prozess der eng und enger zusammenhängenden Ausdifferenzierung ihrer drei dominanten Produktionsformen gekennzeichnet: der politisch-staatlichen, der ökonomisch-kapitalistischen, der individualistisch erwerbs-, besitz- und arbeitsethischen.

Der Typus des modernen Staates entstand hierbei in Form des Monopols legitimer physischer Gewalt. Hobbes hat die Formierung dieses Anspruchs in einer legitimationstüchtigen Fiktion über die Gründe und die Eigenart des Gesellschaftsvertrags kondensiert. Der Staat zeichnete sich, ideengeschichtlich gesäumt, von Anfang an dadurch aus, wenngleich ein langwieriger geschichtlicher Vorgang erst die Sortierung perfektionierte, dass er die Gewalt kumulierte, legitime Gewalt beanspruchte und mittels dieser Gewalt seine nach innen Privilegien schützende und zugleich befriedende Funktion ausübte. Der 
innen gewaltmonopolig durchgesetzte Friede garantierte die Freisetzung der ökonomischen Interessen, die dem Frühliberalismus gemäss prinzipiell grenzenlos Waren handeln, dann erwerben, dann mit Hilfe entsprechender neuer Verhältnisse produzieren durften. Es sei denn sie drehten sich physisch unmittelbar gewaltsam. Als Resultat ergab sich: der Staat war gegen Missbrauchsmöglichkeit der Gewalt und allgemein gegen die politischen „Leidenschaften" zu verfassen. Gewaltenteilig. Mit checks and balances, die die amerikanische Verfassung ausmachen und in den Federalist Papers bis heute unübertrefflich formuliert worden sind. Allein die politische Sphäre war zu verfassen; der fiktive Gesellschaftsvertrag bezog sich allein auf den Staat. Deswegen müsste er korrekter Staatsvertrag heißen. Die ökonomische Sphäre konnte den Frühliberalen gemäss frei gelassen werden, weil sie im Unterschied zu den Leidenschaften durch „rationale“ Interessen gekennzeichnet war, die jedenfalls im Resultat, wie die ökonomischen Klassiker darlegten, dem Gemeinwohl aller dienten (vgl. die hübsche ideengeschichtliche Skizze von Albert O. Hirschman 1977; zum eigenartig verengten und von Anfang an asozialen Rationalitätsbegriff siehe unverändert einschlägig Max Webers Protestantische Ethik, die nicht zuletzt als eine Studie zu verschiedenen Rationalitätsbegriffen und dem Sieg eines einzigen engen, weil ungeheuer abstrakten Rationalitätsverständnisses gelesen werden kann).

Wichtig festzuhalten ist: Gewalt war, den allmählich dominant werdenden Annahmen gemäss, nur im Staat und auf ihn bezogen „beheimatet“. Um seiner Kontrolle willen zu verfassen war darum auch nur der Staat. Die Ökonomie kennzeichnete ideengeschichtlich von Anfang an das, was Isaiah Berlin „negative Freiheit“ genannt hat, die ungebremste individualisierte „Begierde zum Haben und auch zum Herrschen“ (Immanuel Kant).

b) Auch der Staat als Organisation von Gewalt wurde legitimatorisch von Anfang an entproblematisiert. Im Kontext der liberaldemokratischen Verfassungsdiskussion geschah das und geschieht dies bis heute sogar in wachsendem Maße. Legitime Gewalt wurde im Staat gleichsam „aufgehoben“, das heißt im schwäbischen, von Hegel berühmt gemachten Verständnis: Gewalt wird als schlimme, illegitime Gewalt beseitigt und sie wird als legitime Gewalt, die darum fast keine Gewalt mehr ist, bewahrt. So konnte es geschehen, dass Norbert Elias in seinem viel gelesenen Prozess der Zivilisation die Entwicklung der Konzentration von Gewalt beim Staat (also der Monopolbildung) mitsamt ihrem Pendant in der Selbstdisziplinierung der Bürgerinnen und Bürger fast wie einen „reinen“ Fortschritt an Humanität beschrieben hat, ohne je auf die neuerdings anderwärts bekannt gewordenen erheblichen und andauernden „Kollateralschäden“ dieses Prozesses auch nur aufmerksam zu machen. Die Eurozentrik seiner Beobachtungen über die von allen Problemen erleichterten „Verflechtungsprozesse“ - Elias' Ersatzausdruck für Analy- 
se und Zurechnung - verstärkt nur die Camouflage der Gewalt des Staates. Von Kriegen, Expansionen, Imperialismen und Kolonialismen, als handele es sich dort nur um vermeidlich ärgerliche Übergriffe, vielleicht gelesen, aber nicht in Sachen Prozess der Zivilisation beachtet.

Walter Benjamin hat an einem der hauptsächlichen Instrumente des modernen Staates, dem gesatzten Recht - heute nicht weniger gültig, im zunehmend verhunzten, weil die Rechtsform beliebig dehnenden „Rechtsstaat“ eher mehr -, trefflich gezeigt, wie massiv und mit welchen Effekten staatliche Gewalt überall und dauernd anwesend ist:

„Die Funktion der Gewalt in der Rechtsetzung ist nämlich zweifach in dem Sinne, dass die Rechtsetzung zwar dasjenige, was als Recht gesetzt wird, als ihren Zweck mit der Gewalt als Mittel erstrebt, im Augenblick der Einsetzung des Bezweckten als Recht aber die Gewalt nicht abdankt, sondern sie nun erst in strengem Sinne, und zwar unmittelbar, zur rechtsetzenden macht, indem sie nicht einen von Gewalt freien und unabhängigen, sondern notwendig und innig an sie gebundenen Zweck als Recht unter dem Namen der Macht einsetzt. Rechtsetzung ist Machtsetzung und insofern ein Akt von unmittelbarer Manifestation der Gewalt" (Benjamin 1966: 61).

Da dieser wechselseitig konstitutive Zusammenhang, sieht man deskriptiv analytisch nicht weg, nicht in Abrede gestellt werden kann, ist es umso negativ folgenreicher - unter der Perspektive der möglichst weitgehenden Minimierung von inner- und zwischengesellschaftlicher Gewalt gesprochen -, wenn das staatliche Gewaltmonopol, sei's in seinen hauptsächlichen Institutionen, sei's in seinen Funktionen, nicht zuletzt die gesellschaftlichen Bedingungen zu erhalten, die dann privat genannte Gewalt produzieren, sei's als andere Seite der Rechtsetzungsmedaille in Form der Rechtsdurchsetzung mit allen ihren die gesamte Gesellschaft durchdringenden Wirkungen nicht einmal liberaldemokratisch zureichend thematisiert, sondern gleichsam vor die Verfassungsklammer als „staatliche Essenz" schlechthin gezogen wird. Darum sind Rechtsexegesen wie die der bedeutenden Theoretiker Habermas und Luhmann so blindmachend ärgerlich. Diese Rechtsexegesen stellen nichts anderes dar als eine begriffsvergessene „Induktionseselei“ (Friedrich Engels) etablierten opportunistischen Rechtsumgangs (siehe dazu meine längliche Rezension in PROKLA Nr. 94 und 95, 1994).

Genesis und Geltung sind gewiss nicht eins. Man versteht indes die Geltung nicht, wenn man die Genesis auslässt. Beachtet man diese, dann wird die Geltung in ihrer einseitigen Interessehaltigkeit, in dem, was dauernd funktionierend in ihr steckt, in ihren alles andere als selbstverständlichen und so nötigen Institutionen offenkundig. Darum ist daran zu erinnern, dass das staatliche Gewaltmonopol anders als Aphrodite nicht aus der herrlich weißen Schaumkrone klarfrischen Wassers geboren worden ist. Vielmehr ist der Monopolisierungsprozess in Form von Kriegen, bei denen „innen“ und „außen“ meist nicht unterschieden werden konnte, vor sich gegangen. Der Monopolerfolg kam kriegerisch zustande. Das Gewaltmonopol ist mitsamt seinem allgemeingültigen, notfalls vom Monopol erzwungenen Geltungsanspruch gera- 
dezu wörtlich aus Strömen kriegerisch vergossenen Blutes geboren worden (vgl. unverändert trefflich Hintze 1970; und neuerdings Tilly 1992). Es gilt indes nicht nur, dass diese jahrhundertelang bluttriefende Entstehungsgeschichte im nie fertigen, jedoch im 19. Jahrhundert etablierten Nationalstaat aufgehoben worden ist (siehe zur Bedeutung von „aufheben“ oben). Fast noch wichtiger ist die Beobachtung, dass sich das einmal etablierte Gewaltmonopol über seine normale gesellschaftliche Ausstrahlung hinaus dauernd erneuernd bestätigen und also betätigen muss; und dies inmitten seiner nicht zuletzt ökonomisch klassenspezifischen Aufgaben erneut innen- und außengerichtet, wie sie sind.

c) Beschränkte man sich, um herauszufinden, was kapitalistische Ökonomie ist, darauf, die ökonomischen Klassiker und ihre modell- und gesetzestollen und aseptischen Neo-Klassiker zu lesen, man würde vor allem Verständnis und über ein solches hinaus zum Gläubigen gebildet. Als regiere der Markt, von gleichschultrigen Marktteilnehmern in diversen Märkten bevölkert, die mit sich auf gleichem Niveau angesiedelte Kauf-, und Arbeits- und Schuldverträge und ähnliche Verträge mehr eingehen. Staatliche Gewalt „von außen“ ist allein nötig, um Betrüger zu bestrafen, um das von gleich zu gleich wachstumsfördernd hin und her wogende Marktgeschehen zu garantieren, indem die Sicherheit „freien“ Besitzes, „freier“ Verträge und „freier“ Konkurrenz gewährleistet wird. Ansonsten gilt in bewährter Weise die von Adam Smith freilich noch ungleich mehr „moralphilosophisch“ begründete invisible hand. Heute noch verborgener und noch perfekter im hochabstrakten Marktmodell. Letzteres entspricht nur dann nicht „der Wirklichkeit“, wenn es aus irrationalen politischen, beispielsweise auch menschenrechtlichen Gründen gestört wird. Sonst gilt unverändert: Capitalism and Freedom (so Milton Friedman's populäre Propagandaschrift) im Sinne von: capitalism equals freedom, das heißt: it is the precondition and it produces freedom. Darum hat in solcher Perspektive schon der Machtbegriff im Kontext der Ökonomie nichts zu suchen. Gewalt ist ökonomischen Sachverhalten, die nicht als Sozialverhalte verstanden werden, ohnehin fremd. Sie gehört einem anderen, eben dem politisch staatlichen oder terroristischen Stern an. Noch in den unterschiedlichen, hier jedoch konvergierenden System-Begriffen von Luhmann und Habermas wird ohne weitere Analyse behauptet, dass das kapitalistische und das etatistische „System“ in sich sachzwangsartig, ohne spezifische Interessen, ohne Macht- und Gewaltumstände, nicht zu reden von höchst ungleichen Voraussetzungen und Folgen, funktionierten.

d) „Wenn das Geld, nach Augier, ,mit natürlichen Blutflecken auf einer Backe zur Welt kommt', so das Kapital von Kopf bis Zeh, aus allen Poren, blutund schmutztriefend“" (Marx 1867: 788)

Genetische und funktionale Betrachtung lehren allerdings einen vorurteils- 
freieren Blick. Karl Polanyi zeigt in seiner durch viele anderen Quellen zusätzlich belegbaren Studie The Great Transformation, in der die für unsere Zeit endgültige Durchsetzung kapitalistischer Ökonomie im 19. Jahrhundert im europäisch angelsächsischen Umkreis nachvollzogen wird, in welcher Weise die kapitalistische Vergesellschaftung die gesamten bestehenden Vergesellschaftungsformen barbarisierend zerstörte. Außerdem macht er deutlich, was jede einigermaßen balancierte Geschichte bestätigt, wie sehr der kapitalistische Markt von „außerökonomischer" staatlicher Gewalt erzwungen worden ist. Alfred Bürgin kennzeichnet den Vorgang, die Entwicklungen zusammenfassend, als „Enthistorisierung, Enthumanisierung und Entgesellschaftung“ (Bürgin 1993: 15).

Die wohlbelegten Beobachtungen und Argumente der beiden Wirtschaftshistoriker, die den massiven Umfang an oft eingesetzter, jedoch nicht durchgehend nur staatlich geübter Gewalt beim Übergang von der gewiss ambivalenten „Moral Economy“ (E.P. Thompson) vorstellen, werden von Friedrich Engels' nach wie vor lesenswertem Klassiker Zur Lage der arbeitenden Klassen in England unterstrichen und insbesondere im zu Recht berühmten 24. Kapitel von Marx' erstem Kapital-Band - Die sogenannte ursprüngliche Akkumulation - historisch systematisch kondensiert.

Schon zu Beginn warnt Marx vor einem idyllischen, auf Harmonie erpichten Blick. Als habe Gewalt eine vernachlässigbare Rolle gespielt, die man insbesondere, sobald die kapitalistische Ökonomie einmal etabliert worden ist, übersehen könne:

„In der wirklichen Geschichte spielen bekanntlich Eroberung, Unterjochung, Raubmord, kurz Gewalt eine große Rolle. In der sanften politischen Ökonomie herrschte von jeher die Idylle. Recht und ,Arbeit' waren von jeher die einzigen Bereicherungsmittel, natürlich mit jedesmaliger Ausnahme von ,diesem Jahr'.“ (Marx 1867: 742)

Will man Kapitalismus in Theorie und Praxis verstehen, ist es deswegen erforderlich, Geschichte in der Art einer Detektivgeschichte zu treiben, worauf andere als Herrschaftsgeschichte ohnehin allemal hinausläuft. Das heißt: die abstrakte Terminologie, die von gesellschaftlichen Voraussetzungen, Umständen und Konsequenzen absieht - abstrahere heißt von Besonderem absehen oder des Besonderen berauben -, ist auf ihre ausgelassenen Vermittlungen hin aufzubrechen. Die Prämissen der „Gesetze“, die wie solche der Natur behandelt werden, sind zu ermitteln. Dann stellt sich heraus, warum und in welcher Weise vom ersten ungleichen Arbeitsvertrag und ausbeuterischen Arbeitstag an Ungleichheit produziert und die „Ressourcen“ der human „inneren“ und der human „äußeren“ Natur vernutzt, aufgebraucht, erschöpft und zerstört werden. Unter anderem folgende Schritte sind historisch systematisch nachzuvollziehen, um dem human überaus kostenreichen „Modellplatonismus“ (Albert 1969) zu entgehen. 
(1) Die sogenannte ursprüngliche Akkumulation hebt nicht auf dem ebenen Feld sozialer Gleichheit an, auf dem alle Beteiligten auch nur chancengleich antreten. Sie beginnt inmitten einer Struktur der Ungleichheit.

(2) Die „ursprüngliche“ Akkumulation ist nicht ein für allemal beendet. Sie beginnt inmitten einer noch umfassenderen Struktur der Ungleichheit in ungleich rascherer Zerstörung im Verlauf der kapitalistischen Expansion über die europäisch angelsächsischen Ursprungsländer hinaus.

(3) Die strukturelle Ungleichheit und ihre immer erneute Produktion und Reproduktion besitzen zwei immanente Gründe: zum ersten die prinzipielle Besitz- und später Verfügungsteilung zwischen den Besitzern/Verfügern der Produktionsmitteln und denjenigen, die im zeitgenössischen Euphemismus ihre Arbeit „nehmen“. So stark dieser Unterschied im Laufe der Zeit modifiziert worden ist, Marx selbst hat dies im 3. Band des Kapital angesichts des Aufkommens der Aktiengesellschaften schon beobachtet, so orientierungs-, verhaltens- und nicht zuletzt herrschaftsmächtig ist er doch geblieben. Der andere immanente Grund ist in der Konkurrenz gegeben, die sich auf verschiedenen Ebenen und zwischen höchst ungleichen Konkurrenten abspielt mit radikal verschiedenen Mitwirkungsoptionen oder der Option, einen anderen Wirkungsbereich zu suchen (vgl. Hirschman 1970). Diese Konkurrenzen produzieren dauernd Gewinner und Verlierer, sie produzieren gleichfalls andauernd Menschen, die aktuell „gebraucht“ werden und solche, die, als „sozialer Schrott“ beiseitegeschoben, allenfalls noch polizeiliche Ordnungsaufgaben darstellen. Etwa um die Innenstädte der Habenden von den verarmten Nichthabenden frei zu halten.

(4) Die Vorstellung des „Marktes“, mit der die Annahme ebenerdiger unmittelbarer Austauschakte von Gleich zu Gleich verbunden ist, ist längst zu einer schlechten, nämlich fehlleitenden Fiktion geworden. Der Modell-Markt wird von der ökonomischen „Theorie“ durch die bekannten Annahmen konstruiert: ceteris paribus, also wenn alle anderen Faktoren stillgestellt werden, und rebus sic stantibus, wenn sich nichts weiter verändert; er unterstellt eine strukturelle Gleichheit derjenigen, die am Markt teilnehmen; gleichfalls wird angenommen, alle könnten genügend informiert teilnehmen. Der „Markt“ ist stattdessen der längst nicht mehr physisch fassbare „Ort“ eines globalen Machtspiels höchst ungleichgewichtiger Teilnehmer auf unterschiedlichen Ebenen der Konkurrenz. Die sogenannten externen Effekte sind nicht nur und nicht einmal primär umweltbezogen zu beobachten. Sie zeigen sich vor allem, selbstredend kapitalismusintern produziert, in der abgestuften Marginalisierung umfänglicher Teile der Bevölkerung. Deren „negative“ Konkurrenz unter einander bis hin zu den Gruppen, die gänzlich „herausfallen“, besorgen eine Art „negative“ Kooptation.

(5) So wie Kapitalismus bis hin zur Globalisierung und punktuell erreichten Globalität dadurch gekennzeichnet ist, dass er Räume, dass er Zusammen- 
hänge dehnt und Zeiten bis zur ihrer schieren Aufhebung verkürzt - darum sind die Verkehrs- und Kommunikationstechnologien seit der Erfindung der Eisenbahn die führenden Branchen -, so besteht, im Oxymoron gesprochen, seine soziale Produktionsweise in der Dissoziation. Dieses kapitalistische „Gesetz“ der Auflösung gesellschaftlicher Zusammenhänge, das von der staatlich bewirkten Dissoziation begleitet und ergänzt wird, wirkte historisch überaus ambivalent. Auf der einen Seite befreiten staatlich-kapitalistisch bewirkte Dissoziationen in Richtung „ungeselliger Geselligkeit“ (Kant) aus allen Arten feudal enger Verstrickungen. Erst jetzt wird ein Freiheitsbegriff möglich, der räumlich soziale Freizügigkeit, also auch eine gewisse Abstraktion vom „Terror konkreter Bindungen“ voraussetzt. Auf der anderen Seite sind die Kosten der diversen Bauern- und Arbeiter-"Befreiungen“ und der „Befreiungen“ zuerst kolonialisierter, dann unter die „Marktgesetze“ gezwungener Gesellschaften nicht nur vorübergehend ungeheuer. Diese Feststellung gilt vor allem deswegen, weil keine neuen verbindlich partizipativen sozialen Formen und ihnen gemäße Orientierungen an ihre Stelle treten. Darum bedeuten die kapitalistischen Dissoziationen, die in der Zwischenzeit alle anderen Vergesellschaftungsformen zerstört haben, selbst in den Ländern, die sich jahrhundertelang zur kapitalistisch nationalstaatlichen Gesellschaft entwickeln konnten, also den europäisch angelsächsischen, weithin Formen der Vereinzelung, die nicht positiv im Sinne orientierungs- und handlungsfähiger Individuen wahrgenommen werden können. So tun dies geradezu analysefrei und geradezu grotesk anti-soziologisch Ulrich Beck und seine kollektiv der Individualisierungsthese folgende nachplappernde Meute. Schein wird postmodern Sein. Längst wären die von Lukacs und anderen weiterentwickelten Marxschen Termini wieder erneuernd und angemessen modifizierend aufzugreifen, die tief in der Aufklärung und ihrem „Menschenbild“ ankern. Sie sind mit der Beerdigung der Studentenbewegung und der damit nolens volens gekoppelten Kritischen Theorie wenn nicht in Verruf, so doch in Vergessenheit geraten. Als da sind: Verelendung, Entfremdung, Verdinglichung, Emanzipation u.ä.m. Zur Illustration eigneten sich neuerdings Richard Sennetts Flexibler Mensch und eine Fülle betriebssoziologischer Studien. Deren Ergebnisse müssten allerdings mit kritischeren Augen gelesen werden als die vielen, eher affirmativen Studien zur angeblich neuen Gruppenarbeit, zu den behaupteten abgeflachten Hierarchien, zur partizipativen Innovation, zur angeblichen „Humanisierung der Arbeitswelt" im allzu rasch als dominant behaupteten höchst ambivalenten „Postfordismus“.

All diese flächig erinnerten Facetten kapitalistischer Entwicklung und Gegenwart unterstreichen in dem hier thematisierten Zusammenhang ein Dreifaches: zum einen in welch enormem Umfang die kapitalistische Vergesellschaftung die Gesellschaften und die in ihr lebenden Menschen formt. Darum ist das Als-Ob der angeblich wissenschaftlichen Ökonomie, sie sei eine 
Gesetzeswissenschaft von „Ökonomie pur“ so radikal falsch und zugleich so voll negativer Effekte; zum anderen gilt, dass die Formierung der Menschen und ihre Möglichkeiten durch den Kapitalismus alles andere bewirkt als selbstständige und handlungsfähige Personen, das Zielbild der frühliberalen Aufklärung. Die a-sozialen Interessen werden enorm verstärkt. Damit verbunden die Abhängigkeiten, die Ängste, die Vorurteile; das dritte Merkzeichen kapitalistisch fortschreitender Vergesellschaftung sind in allen Ländern, am meisten in solchen eher ,junger“, meist segmenteller und insularer Durchkapitalisierung, gesellschaftliche Zwänge zur anhaltenden Mobilität inmitten der sozialen Trümmer nicht mehr funktionsfähiger früherer Vergesellschaftungsformen. Diese „objektiven“ Zwänge sind nicht selten mit verelendendem, ja mit tödlichen Ausgang verbunden.

e) Die Entwicklungen des modernen Staates und der modernen Kapitalismus sind vielfach, zuweilen bis zur Unkenntlichkeit miteinander verschlungen. So sehr für die Anfänge beider Vergesellschaftungsformen mehrere, zum Teil unterschiedliche Entstehungsgründe angenommen werden können, so sehr gilt, dass beide Vergesellschaftungsformen, je mehr sie sich als die gesellschaftlich dominanten etablierten, desto mehr auf einander angewiesen waren. Ihre institutionelle und funktionale Differenz machte sie im Prozess der Durchsetzung und Expansion wechselweise in zunehmendem Maße von einander abhängig. Ohne die staatliche Gewalt wären die „freien“ Bedingungen kapitalistischer Ökonomie nicht zu schaffen gewesen. Vor allem fehlte gerade in den kurzzeitigen ökonomischen Mikro- und Makrozyklen die kalkulierbare, in diesem Sinne rationale institutionell garantierte Dauer. In diesem Sinne gilt: keine (kapitalistische) Rationalität ohne (in der Regel: staatliche) Gewalt. Die angewandte Abstraktion als welche die Formen kapitalistischer Ökonomie gegen alles, was gesellschaftlich vorher war, bis heute eingeführt und durchgesetzt werden, wäre ohne staatlichen Zwang nicht erfolgreich gewesen, von der nicht endenden Geschichte kapitalistischer, militärisch begleiteter Expansionen nicht selten mit massenmörderischem Ausgang gar nicht zu reden. Die staatlich legitimatorische Normalisierung, ja Adelung des Krieges ist ein Sonderthema. Die nationalsozialistische Großraumpolitik mitten im Krieg und durch den entgrenzenden Krieg stellt ein einseitiges Extrem dar, indes ein Extrem kapitalistisch etatistischer „Normalität“.

Die doppelte Gewalt-Genese staatlicher und kapitalistischer Etablierung, die sich in veränderter Form fort und fort wiederholt, bildet nur die eine Seite. Die andere zeigt sich, wenn man alle hauptsächlichen staatlichen und kapitalistischen Institutionen und Funktionen Revue passieren lässt und sie in ihrer Differenz, jedoch zugleich in ihrem beiden Vergesellschaftungsformen notwendigen Zusammenhang betrachtet. Dann wird einsichtig, dass staatliche Gewalt ohne die schmale Rationalität kapitalistischer Vergesellschaftung, je- 
denfalls in der im Laufe des 19. Jahrhunderts durchstaatlichten Massengesellschaft nicht funktionstüchtig wäre. Dann wird gleicherweise kenntlich, dass die kapitalistische „Rationalität“ ohne staatliche Gewalt ihrerseits nicht funktionieren könnte. Mit anderen Worten: nicht nur der Staat ist unverhüllt von seiner oft gewalttätig errungenen allgemeinen Legitimation als eine Gewaltinstanz in all seinen Institutionen, Funktionen, Wirkungen und Kosten zu analysieren. Auch die kapitalistische Vergesellschaftungsform kann nur zureichend beschrieben und erkannt werden, wenn man sie mit Hilfe von Machtund Gewaltbegriffen beschreibt und analysiert. Hierbei ist erneut das wechselweise Spiel der beiden schlechterdings dominanten Vergesellschaftungsformen zu beobachten, sich zu konstituieren und zu legitimieren. Dieses Wechselspiel trägt vielfach zur perspektivischen Täuschung bei bis hin zum Als Ob ausdifferenzierter Entitäten hier „Staat“ (Politik), dort „Kapital“ (Ökonomie) (gar als spheres of justice von Michael Walzer u.a. überhöht). Mit Hilfe seiner eigenartigen Ressource allgemeiner Legitimation - sie kennzeichnet staatliche Politik mitsamt seiner anderen eigenen Ressource, dem Gewaltmonopol als potentiell eigensinnig - rechtfertigt der Staat funktional hauptsächlich, übersetzt von all seinen wichtigsten Institutionen und Instrumenten, das ökonomisch Besondere, also - mit dem euphorisch euphemistischen Ökonomen Recktenwald gesprochen - die „tausendfältigen Spontaneitäten des Marktes“. Das politisch staatlich Allgemeine schafft, schützt und schirmt das Besondere kapitalistischer Interessen und deren Freizügigkeit. Das war, ist und bleibt der Kern aller liberaldemokratischen Theoreme und Praktiken. Das heißt zugleich: die der kapitalistischen Gesellschaft eigene Struktur ungleicher Gesellschaft national und international/global ungleicher Gesellschaften, recht verstanden also die Klassenspezifik der Gesellschaften wird mit Hilfe der allgemeinen, prinzipiell alle Bürgerinnen und Bürgern einbeziehenden Legitimation immer erneut bestätigt und notfalls gewaltsam bewahrt bzw. erneuert. Das Allgemeine ist also, im praktizierten Paradoxon, das Besondere. Dem dient auch nach innen und außen letztlich und erstlich das allgemein legitimierte Gewaltmonopol. Darum ist auch die „Legitimation durch Verfahren" (Niklas Luhmann), sind also die wahlzirzensischen symbolic uses of politics (Murray Edelman) so wichtig.

Umgekehrt ist das Allgemeine, also staatliche Politik nicht „lebensfähig“ ohne die materiell-fiskalischen Ressourcen aus der Ökonomie, an deren Wachstum staatliche Politik schon deswegen brennend interessiert sein muss. Die dissoziative Wirkung kapitalistischer Vergesellschaftung und ihre Vereinzelung in Bewusstsein und Verhalten bis hin zum alles orientierenden shareholder value tun ein Übriges um staatlich ihrerseits abstrakt gewordene Herrschaft entgegen aller störenden Assoziationen zu stabilisieren.

Schon vor aller globalisierenden Ver-Rückung der ungleich zusammengehörigen Tandemradler „Politik“ und „Ökonomie“ ist also das bestimmende Pri- 
mat der Ökonomie, ihrer Struktur der Privatheit und ihrer interessenschmalen Orientierung und Motivierung gegeben. Das, was ökonomisch massiv an Dauergewalt und hin und wieder aktueller Gewalt bedarf, wird soweit wie möglich legitimatorisch dem Staat abgegeben, der die funktional dem Besonderen dienende Gewalt allgemein rechtfertigt. Umgekehrt können strukturelle und aktuelle Ungleichheiten staatlich gleichsam an die Ökonomie abgeschoben werden. Letztere funktioniert angeblich nach ganz eigenen Gesetzen. Staatliche Einmischung würde deren wohlständische „Effektivität“ gefährden. Bestenfalls sind je nach Konjunktur- und weltmärktlicher Konkurrenzlage soziale Zwischenpolster, ihrerseits arbeitsmarktkonform einseitig, möglich.

\section{Globalisierung und Gewalt}

Globalisierung mindert die gewaltförmigen Folgen der Kapitalisierung auf neuer Stufenleiter eines ex- und intensiver konkurrierenden Kapitalismus nicht; Globalisierung erzeugt in ihrer Eigenart als dynamisch erneuernd fortgesetzte Struktur der Ungleichheit auf Weltebene die Aggressionspotentiale der Länder und Bevölkerungsteile im Wachstums- und Wohlstandsschatten wie sie die Gewaltpräparationen der Länder fördert, und gegebenenfalls deren so oder so geartete „humanitäre“ oder antiterroristische Interventionen, die sich herrschafts-, sprich zugleich militärstark in der Wachstums- und Wohlstandssonne ihrerseits ungleich sonnen.

„Im Fortgang der kapitalistischen Produktion“, so lautet die wohl bekannteste Stelle des 24. Kapitels des Kapitals, „entwickelt sich eine Arbeiterklasse, die aus Erziehung, Tradition, Gewohnheit, die Anforderungen jener Produktionsweise als selbstverständliche Naturgesetze anerkennt. Die Organisation des ausgebildeten kapitalistischen Produktionsprozesses bricht jeden Widerstand, die beständige Erzeugung einer relativen Überbevölkerung hält das Gesetz der Zufuhr von und Nachfrage nach Arbeit, und daher den Arbeitslohn, in einem den Verwertungsbedürfnissen des Kapitals entsprechenden Geleise, der stumme Zwang der ökonomischen Verhältnisse besiegelt die Herrschaft der Kapitalisten über die Arbeiter. Außerökonomische, unmittelbare Gewalt wird zwar immer noch angewandt, aber nur ausnahmsweise. Für den gewöhnlichen Gang der Dinge kann der Arbeiter den 'Naturgesetzen der Produktion' überlassen bleiben, d.h. seiner aus den Produktionsbedingungen selbst entspringenden, durch die garantierte und verewigte Abhängigkeit vom Kapital. Anders während der historischen Genesis der kapitalistischen Produktion.“ (Marx 1867: 765)

Trotz der enormen Entwicklungen, Veränderungen und Brüche seither welch eine Klarsicht. Welche in den wichtigsten Formulierungen schier unmittelbare heutige Geltung. Im Jahr 2001, nicht nur 1867. Es wäre überaus lohnend, so präzise wie möglich herauszuarbeiten, was warum seither prinzipiell gleich geblieben ist, was warum sich prinzipiell verändert hat - das kann hier nicht geschehen. Ich will nur auf einige Entwicklungslinien des Kapitalismus im Zeitalter seiner globalisierten und sich weiter, höchst widerspruchsvoll globalisierenden Entpuppung hinweisen, die Marx nicht in allen, jedoch in entscheidenden Aspekten vorhergesehen hat, angefangen von dem heute trotz riesiger humaner und ökologischer Kosten mehr denn je geltenden, scheinbar alternativlosen stummen Zwang kapitalistischer Verhältnisse und ihrer 
naturgesetzlichen Geltung. Und dieser Zwang, das macht gerade seine Gültigkeit, seine Legitimiertheit ohne Legitimation aus, spricht seine systemische, seine politisch begründende und flankierende Gewalt eben nicht aus.

Auf die Kennzeichen des globalen, sich globalisierenden Kapitalismus im Sinne der weiteren und sich dementsprechend verändernden Realisierung dessen, was im Begriff des „Kapitals im Allgemeinen“ steckt als einem historischen Kondensat interessenstark miteinander verschlungener sozialer Verhältnisse, werfe ich nur einige nicht weiter belegte Schlaglichter:

- die Gleichschaltung der von Menschen bewohnten Welt, ja sogar des noch unbesiedelten Raumes im Sinne eines Interesses, das jedoch nur in erneuerter Ungleichheit wahrgenommen werden kann. Das Ausfällen ganzer „Menschentypen " und Gruppen von Menschen gehört dazu;

- die radikale, sprich zur Funktionswurzel kapitalistischer Vergesellschaftung gehörige Produktion von Ungleichheit. Diese strukturelle Ungleichheit wird funktionell durch die Eigenart kapitalistischen Fortschritts erneuert. In der „Begierde zum Haben“, um die gelungene Kant'sche Formulierung noch einmal aufzugreifen (siehe Idee zu einer allgemeinen Geschichte in weltbürgerlicher Absicht), steckt von allem Anfang an, als kapitalistisches Alpha und Omega, die von Kant gleichfalls gesehene und nur durch die Koppula „und auch“ verbundene und zugleich hervorgehobene „Begierde zum Herrschen“. Darum auch, aber nicht nur das militärische Schwert und der militärische Schild. Man höre nur, eine beliebige Illustration, den O-Ton des Herrn Schröder, seinerzeit Bundeskanzler der BRD unter anderem am 16. November 2001, einem Tag, an dem sich der Bundestag vollends den exekutivisch vorgesagten „Sachzwängen“ unterwarf (dazu noch weiter unten): zur erneuernden Erhaltung des Wohlstands gehöre Weltpolitik; und zur Weltpolitik gehöre nun einmal Militär; und zum Militär selbstredend, dass man es bei gegebener Gelegenheit auch einsetzt;

- die anhaltende Sozialgeschichte kapitalistischer Befreiungen in Form einer weltweiten Binnenmigration in einzelnen Ländern und einer noch erheblicheren Migration zwischen den Ländern und Länderblöcken. Und in dieser vielfältig motivierten Migration gerade auch der bundesdeutsch gerichtsnotorisch diskriminierten „Wirtschaftsflüchtlinge“ steckt Gewalt;

- die anhaltende, nun weltweit gedehnte Abstraktion. Diese Abstraktion ist verbunden mit schier unübersehbaren Größenordnungen: von Menschen, ihren Interessen, von Waren, von Geldströmen vor allem, von Profiten und ineins damit von schon allein durch ihre Größenordnungen mächtigen Institutionen, die als diverse global and regional players in Erscheinung treten. Die Beschleunigung der Kommunikationen vergrößert ihrerseits die alles andere als statischen Größenordnungen zum schlechterdings nicht mehr Fassbaren. Die von diesen Größenordnungen bewirkten Effekte bestehen zu allererst darin: dass Besonderheiten nicht zählen. Das hebt global-oben an und 
hört lokal-unten auf. Denn Lokalitäten werden gerade in ihren besonderen, sprich eigensinnigen Bedingungen aufgelöst. Die immer geltende, seit dem 11. September besonders anschauliche Rolle des Unanschaulichen, also die Geopolitik verbunden mit der Geoökonomie - man denke hier nur an das EU-Europa und sein strukturell unvermeidliches Demokratiedefizit - und dem Geomilitär bieten dafür die besten und also die menschenrechtlich demokratisch schlimmsten Belege. Diese Art der Globalisierung fördert die Menschenrechte nicht, sie entspricht den Menschenrechten nicht, sie widerspricht denselben frontal, so Menschenrechte die Lebensrechte aller Menschen als je historisch kulturell besonderen Wesen bedeutet;

- die Orientierungs- und die Organisationslosigkeit, so man unter letzterer soziale Einrichtungen versteht, die es ermöglichen, die von ihnen ausgehenden Handlungen intern und extern prinzipiell verantwortlich zu kontrollieren.

All diese und zusätzliche wichtigen Merkmale dessen, was da ökonomisch technologisch von oben, also abstrakt bestimmt, hochgradig ungleich realisiert und realisierbar Globalisierung heißt, schürzen sich zu Bedingungen, die eine Weltlandkarte der Verelendung, der Orientierungslosigkeit, des Mangels zuverlässiger Organisationen zeichnen ließen. Diese Landkarte wäre zu ergänzen durch eine der Wachstumsregionen, der Habenden verbunden mit den Verelendenden unter den Habenden - man betrachte allein das reichste Land der reichen Länder, die USA oder auch in kleineren Dimensionen die BRD. Beide Karten wären zu ergänzen durch die Geoökonomie und Geopolitik der Interessenherrschaft, die die Habenden über die Nichthabenden national, vor allem jedoch weltweit ausüben.

Diese schwierigen, weil komplizierten Karten, die jedoch in behutsamer Simplifizierung gezeichnet werden könnten, ja müssten, wären nicht dichotomisch anzulegen, geteilt in die Habenden und die Nicht-Habenden und ihre jeweiligen Herrschaftsinstrumente. Gerade die Staaten und ihre Ökonomien dazwischen müssten berücksichtigt werden, um das herauszufinden, worum es hier geht: Gewaltpotentiale und ihre Aktualitäten mehrfacher Art, die miteinander Gewalt-konkurrieren. Die einen Orwell gemäß mit ungleich mehr Gewalt, die anderen mit bei weitem weniger. Die Gewalt, die als mehr oder minder stummer Zwang in der nicht zuletzt kapitalistisch etatistisch seit Kolonialisierungszeiten bewirkten Zerstörung und Verelendung steckt, muss ebenso zum Sprechen gebracht werden, wie die Vorbereitungen, sich gewalttätig zu „emanzipieren“ von Gruppen und Staaten oder Quasistaaten, die nicht zu den Habenden, jedoch auch nicht zu den ganz verelendeten gehören. Schließlich ist die versammelte Gewalt zu untersuchen, die die HabendHerrschenden Länder samt ihren global expandierten Global Players ausüben, nicht selten vermittelt durch die internationalen Einrichtungen von der Weltbank bis zur WTO, die ihrerseits „westlich-nördlich“ dominiert werden. 
Erst solche skrupulösen Klärungen, informiert von einem materialistischen Begriff der Menschenrechte, der darum das je Besondere nicht dem abstrakt Allgemeinen eines existenzlosen Individuums opfert, erlaubten eine Analyse der entwickelten und andauernden Dialektik von Gewalt auf globaler Stufe mit der Chance kognitiv analytisch zureichend informiert, die Ursachen der Gewaltspiralen des 21. Jahrhunderts zu bekämpfen.

\section{Der 11. September und seine Folgen, eine verkürzte aktuelle ,Nutzanwendung'}

Das, was Arundhati Roy und andere nach dem 11.9. 2001, einem schlechterdings nicht rechtfertigbaren terroristischen Akt ausgedrückt hat, bestimmt den seitherigen Skandal nach der inszenierten Katastrophe, ein Skandal der die nächsten Katastrophen produziert. Dass bei denjenigen, die angeblich die Opfer repräsentieren, die angeblich die Opfer rückwärts sühnen und vorwärts verteidigen wollen, indem sie nächste Opfer mit allen möglichen Mitteln vermeiden wollen, nicht nur die Ursachen, die zum 11. September führten, seine unmittelbaren und vor allem seine mittelbaren Gründe nicht interessieren. Vielmehr kümmern die vereinten Regierungen der NATO-Staaten, die US-Regierung an abständiger Spitze auch die Menschen nicht, die von ihren angeblich antiterroristisch ausgerichteten Aktionen betroffen und getötet werden. Von selbst versteht es sich schon, dass die Folgen der eigenen Folgerungen, also der kriegerischen Aktionen über die unmittelbaren Effekte hinaus nicht kümmern. Dass im Gegensatz zu den Behauptungen die Gewaltspirale kräftig angetrieben wird.

Diese Missachtung des Besonderen, der Menschen an ihren besonderen Orten mit ihren Eigenarten ist es, was die hochgehaltene westliche Zivilisation genetisch und funktional inmitten ihres an sich schönen Begriffs und der an sich schönen Sache der Zivilität so blutig macht und blutig hält. Ich will an den anderen Prozess der Zivilisation, an die Geschichte der Eroberungen, der Kolonialisierungen, der Kolonial- und Postkolonialkriege nicht weiter erinnern. Ich will auch nicht spezifischer daran erinnern, dass die Intoleranz gegen anders, also nicht kapitalistisch-etatistisch Lebende die westliche Zivilisation von allem Anfang an auszeichnet und darum genozidale Spuren in der Art dieser Zivilisation bis hin zu ihrem frühen Menschenrechtsbegriff enthalten sind. Das nicht zur Kenntnis zu nehmen, nicht die Arbeit begrifflich praktischer Erneuerung zu leisten, das ist in der Tat Schuld. Und diese Schuld wird dann „praktisch“ in neuen ungenannten humanen Kosten, die die sogenannte Modernisierungstheorie samt ihrer nicht selten kriegerischen Umsetzung, die auch noch nicht wenige sogenannte Transformationstheorien erzeugen. Dass Pflanzen- und Tierarten aussterben gemacht werden, ist schlimm genug. Dass jedoch ganzen Menschengattungen, mit Max Weber 
gesprochen, Menschentypen das Lebensrecht abspenstig gemacht wird, abgegraben, abgewaldet, wegmodernisiert, das ist unsäglich.

Ich will aktuell aus gegebenem Anlass allein an die Geschichte der letzten 10 Jahre und der in diesem Zeitraum vom „Westen“ „solidarisch“ geführten Kriege erinnern. So unterschiedlich sie sind, sie zeigen jeweils drei sich überdeckende Merkmale: zum ersten, dass die Situation, deretwegen „der Westen“ eingegriffen hat, von eben diesem „Westen“ zuvor wesentlich mitproduziert worden ist; zum zweiten, dass die militärischen Schläge nur Menschen getötet haben, die jeweils gegebenen Probleme jedoch mitnichten humaner angehen ließen (neuerdings ist immer wieder davon die Rede, dass es sich um „unschuldige Menschen“ gehandelt habe, als zählen wie immer schuldige Menschen schon gar nicht mehr als Menschen so wie „Schurkenstaaten“ und die „absolut Bösen“, die in ihnen herrschen); zum dritten, dass die Zeiten nach der westlichen „Kriegshilfe“ schlimmer waren als zuvor und die westlichen Mächte nichts anders als ihren Vorstellungsmangel für die lokalen und regionalen Probleme belegten. Kurzum: alle Konflikte der letzten 10 Jahre, an denen „der Westen“ beteiligt war, wurden nicht nur verschlimmert, sie sind westlicherseits dadurch gekennzeichnet, dass sie eine geradezu radikale Missachtung von Land und Leuten demonstrieren. Am deutlichsten gilt diese Beobachtung, die sich durchgehend belegen ließe, in der von den USA mit UNO-Zustimmung und deutscher Hilfe durchgeführten Aktion mit dem geradezu zynischen Euphemismus: „Restore hope“ 1992 in Somalia. Wie Länder mit den angeblich besten Wissenschaftlern und intelligence services der Welt eine Situation wie die in Somalia so vollkommen verkennen können und darum mit ihrer militärischen Intervention noch kläglicher scheitern als anderwärts, ist nur zu erklären, wenn man annimmt, dass die - meist innenpolitisch stark bedingten - Interessen der USA und ihrer Verbündeten samt der ihre erste kleine militärische Feuertaufe im Form eines Tropfens erhaltende BRD nahezu exklusiv zählten. Ob Somalia, ob der Irak, mit dem von den USA lange gepäppelten Saddam Hussein, ob Jugoslawien und ExJugoslawien, ob das Kosovo oder nun Afghanistan - schade für diese Länder und ihre Leute, dass die westlichen Interessen mit den ihren, die ohnehin nicht zählen, nicht übereinstimmen. Selbst noch eine Verfassungsskizze, die das bundesdeutsche Außenministerium für die Zeit nach dem „Sieg“ für Afghanistan ausgearbeitet hat, zeigt herrschaftliche Hybris dieses Westens, dem andere nur gemäss seinen Interessen und seinen stellvertreterpolitischen Vorstellungen zählen. Der Skizze fehlte nur, dass ein Amt zum Schutz der freiheitlich demokratischen Grundordnung in Kabul mit etlichen Zweigämtern übers Land verstreut einzurichten ist.

Warum diese schlimme Ahnungs- und Vorstellungslosigkeit (nota bene auch vieler sozialwissenschaftlicher Theorien, die „weststämmig“ formuliert werden)? 
Liest man wie ich täglich die Frankfurter Allgemeine Zeitung und hört man sich die Staatsmänner des Westens an, dann möchte man glauben, die westlichen Staaten verbunden im Konzert ihrer Weltpolitik seien gestaltungsstark zurückgekehrt. Wenn (Staats-)Sicherheit zum überragenden außen- und innenpolitischen Terminus wird, dann ist selbstredend der Hobbes'sche, der Carl-Schmitt'sche, Freund von Feinden urpolitisch sondernde STAAT gefragt. Indes, ich weiß nicht, ob ich sagen soll, ich fürchte, dieser staatsmännergewaltige Eindruck täuscht. Er täuscht jedenfalls insofern, als mit diesem Eindruck, die Annahme machtvoller politisch staatlicher Gestaltungskraft verbunden wäre. Sehe ich es recht, sind diese militärisch rasselnden und kriegstümelnden Äußerungen und Taten durch die damit beauftragten jungen Soldaten nichts anderes als Ersatzpolitik. Sie sind einzuordnen in das, was oben über das Verhältnis von Staat und Kapital skizzenhaft angedeutet worden ist, nun im Zuge der Globalisierung noch stärker zugunsten kapitalistischer Interessen verrückt, die freilich immer schon Eigeninteressen der Staatsleute sind. Im Sinne gemeinsamen Positionenhaltens, wenn nicht Verbesserns im Konkurrenzkampf um die Weltmarktmacht. Dieses Interesse ist das, was den „Westen“ „solidarisch“ zusammenhält. Und die militärische Umrüstung, die militärischen Einsätze und die geoökonomisch informierte Geopolitik dienen an erster Stelle dazu, die Weltmarktmachtpositionen zu halten, ja in erwartbar schwierigeren Zeiten zu verbessern. Dafür ist die Legitimation mit dem „Krieg“ gegen den Terrorismus ein gefundenes Fressen. Da das, was Terrorismus ist, ähnlich dem Sackbegriff „Organisierte Kriminalität“ täglich neu werden kann, da das, was jeweils darunter zu verstehen ist, nicht aufhören, ja eben solchen Antiterrorismus kräftig mitproduzieren wird, wird es an Legitimationsbehauptungen für alle möglichen Einsätze, zuweilen auch wieder „humanitär“ gesüßt, nicht mangeln.

\section{Nicht ganz unzusammenhängende Endnote}

Eric Hobsbawm schreibt gegen Ende seines bedeutenden Buches über das 20. Jahrhundert The Age of Extremes:

„In short, the century ended in a global disorder whose nature was unclear, and without an obvious mechanism for either ending it or keeping it under control.“

Die „Natur“ der globalen Unordnung scheint mir nicht ganz so unklar. Ihre Klärung muss m.E. in oben angegebener Richtung erfolgen. Wohl aber trifft zu, dass weltweit ein katastrophenhaltiger Mangel einigermaßen verantwortlicher und also kontrollierbarer Institutionen besteht. Derselbe hebt auf lokaler und regionaler Ebene an, gilt selbst für die einigermaßen verlässlich etablierten Nationalstaaten wie die „westlichen“ und setzt sich auf der internationalen oder auch transnationalen Ebene fort. Diesem Mangel konzeptionell, institutionell und verfahrensförmig in Richtung einer vielfältigen und je ei- 
genständigen Pluralität abzuhelfen - das ist heute und morgen die größte, die schwierigste Aufgabe. Zu ihr gehört an erster Stelle, das zeigt auch diese Skizze, eine erhebliche, wenn nicht radikale Veränderung des „westlich“ dominierten Kapitalismus mit seinen etatistisch-militaristischen Zähnen. Wer Gewaltverhältnisse minimieren will, wer die Zuflüsse zu den Gewaltreservoiren dieser Welt drosseln oder wenigstens kanalisieren will, muss, so meine ich, kräftigen, nicht gewaltigen Schritts in diese Richtung gehen. Gewaltfrei gibt es keine andere.

\section{Literatur}

Benjamin, Walter 1966: Zur Kritik der Gewalt, in: ders.: Angelus Novus, Frankfurt/M. S.42-66.

Bürgin, Alfred, 1993: Zur Soziogenese der Politischen Ökonomie. Wirtschaftsgeschichtliche und dogmengeschichtliche Betrachtungen, Marburg/Lahn.

Hintze, Otto, 1970: Staatsverfassung und Heeresverfassung, in: Ders.(hrsg. von Gerhard Oestreich): Staat und Verfassung, Göttingen, S.52-83.

Marx, Karl, 1867: Das Kapital. Kritik der politischen Ökonomie Bd.1, MEW, Bd.23.

Narr, Wolf-Dieter, 1994: Recht-Demokratie-Weltgesellschaft. Zur Rechtstheorie von Habermas und Luhmann, in: PROKLA Nr.94 und 95.

Polanyi, Karl, 1978: The Great Transformation. Politische und ökonomische Ursprünge von Gesellschaften und Wirtschaftssystemen, Frankfurt/M.

Sofsky, Wolfgang (2001): Der Zerstörte Fluss der Zeit. Urplötzlich bricht die Gewalt herein, ohne jede Vorwarnung: Strukturmerkmale des Terrors als einer universalen Technik der Machtund Gemeinschaftspolitik, in: Frankfurter Rundschau Nr.260 vom 8.11.2001, S.19

Tilly, Charles, 1992: Coercion, Capital, and European States, Blackwell London/New York. 